\title{
ELLIPTIC EXTENSIONS IN THE DISK WITH OPERATORS IN DIVERGENCE FORM
}

\author{
ORAZIO ARENA and CRISTINA GIANNOTTI ${ }^{凶}$
}

(Received 27 May 2012; accepted 2 July 2012; first published online 20 August 2012)

\begin{abstract}
Let $\varphi_{0}$ and $\varphi_{1}$ be regular functions on the boundary $\partial D$ of the unit disk $D$ in $\mathbb{R}^{2}$, such that $\int_{0}^{2 \pi} \varphi_{1} d \theta=0$ and $\int_{0}^{2 \pi} \sin \theta\left(\varphi_{1}-\varphi_{0}\right) d \theta=0$. It is proved that there exist a linear second-order uniformly elliptic operator $L$ in divergence form with bounded measurable coefficients and a function $u$ in $W^{1, p}(D), 1<p<2$, such that $L u=0$ in $D$ and with $\left.u\right|_{\partial D}=\varphi_{0}$ and the conormal derivative $\partial u /\left.\partial N\right|_{\partial D}=\varphi_{1}$.

2010 Mathematics subject classification: primary 35D30; secondary 35J15.

Keywords and phrases: elliptic equations in divergence form, Cauchy data.
\end{abstract}

\section{Introduction}

Let $D$ be the unit open disk in $\mathbb{R}^{2}, n$ the outer normal to $\partial D$ and $L$ a linear second-order uniformly elliptic operator, with bounded measurable coefficients in $D$, of the form

$$
L:=a^{11} \partial_{11}+2 a^{12} \partial_{12}+a^{22} \partial_{22}
$$

In [1], Manselli and the second author proved that, given two arbitrary functions $f^{(0)}, f^{(1)}$ on $\partial D$ (with some appropriate regularity assumption, such as $d f^{(0)} / d \theta$ and $f^{(1)}$ Hölder continuous with exponent $\eta>\frac{1}{2}$ ), there exist an operator $L$ of the form (1.1) and a function $u \in W^{2, p}(D), 1<p<2$, satisfying

$$
\left\{\begin{array}{l}
L u=0 \quad \text { in } D \\
\left.u\right|_{\partial D}=f^{(0)}, \\
\left.\frac{\partial u}{\partial n}\right|_{\partial D}=f^{(1)}
\end{array}\right.
$$

Such a pair $(u, L)$ was called an elliptic extension of $f^{(0)}, f^{(1)}$ in $D$.

Here we consider the following similar question for elliptic operators in divergence form. Given two functions $\varphi_{0}$ and $\varphi_{1}$ on $\partial D$, do there exist a function $u$ and a linear

(C) 2012 Australian Mathematical Publishing Association Inc. 0004-9727/2012 \$16.00 
second-order uniformly elliptic operator $L$ in divergence form, such that

$$
\left\{\begin{array}{l}
L u=\partial_{i}\left(a^{i j} \partial_{j} u\right)=0 \quad \text { a.e. in } D \\
\left.u\right|_{\partial D}=\varphi_{0} \\
\left.\frac{\partial u}{\partial N}\right|_{\partial D}=\varphi_{1},
\end{array}\right.
$$

where $\partial u /\left.\partial N\right|_{\partial \Omega}=a^{i j} u_{x_{j}} n_{i}$ is the conormal derivative of $u$ ?

Wolff [3] studied this problem on smoothly bounded domains $\Omega \subset \mathbb{R}^{n}, n \geq 3$. He proved that in order to have a solution with $u$ and $L$ smooth, the functions $\varphi_{0}$ and $\varphi_{1}$ must satisfy suitable necessary and sufficient compatibility conditions. He also remarked that, for the case $n=2$, additional assumptions on $\varphi_{0}$ and $\varphi_{1}$ are required.

Here, using the result in [1], we prove that, if $\varphi_{0}$ and $\varphi_{1}$ are regular functions on $\partial D$ such that

$$
\int_{\partial D} \varphi_{1} d s=0 \text { and } \int_{\partial D} n_{2}\left(\varphi_{1}-\varphi_{0}\right) d s=0,
$$

then there exist an operator $L$ in divergence form, with bounded measurable coefficients, and a function $u$ in $W^{1, p}(D), 1<p<2$, which satisfy (1.2) in a suitable weak sense.

\section{The main result}

Consider the problem (1.2), where $L$ is a linear second-order uniformly elliptic operator in divergence form, with bounded measurable coefficients, and $u$ is a function in $W^{1, p}(D), p \geq 1$. Notice that, due to the low regularity of $u$ and $L$, the conditions of (1.2) have no meaning, unless they are reinterpreted in a weaker sense, which we now specify. Recall that, if $\Omega$ is a bounded domain in $\mathbb{R}^{2}$, a function $u$ in $W^{1,2}(\Omega)$ is considered a solution of the problem

$$
\left\{\begin{array}{l}
L u=\partial_{i}\left(a^{i j} \partial_{j} u\right)=0 \quad \text { a.e. in } \Omega \\
\left.\frac{\partial u}{\partial N}\right|_{\partial \Omega}=\varphi_{1}
\end{array}\right.
$$

if it satisfies the identity

$$
\int_{\Omega} a^{i j} \partial_{j} u \partial_{i} \eta d x=\int_{\partial \Omega} \varphi_{1} \eta d \sigma \quad \text { for any } \eta \in W^{1,2}(\Omega)
$$

(see, for example, [2, p. 161]).

By analogy with such interpretation, in the following a function $u \in W^{1, p}(D), p \geq 1$, will be considered a solution of (1.2) if

$$
\left\{\begin{array}{l}
\int_{D} a^{i j} \partial_{j} u \partial_{i} \eta d x=\int_{\partial D} \varphi_{1} \eta d \sigma \quad \text { for any } \eta \in C^{1}(\bar{D}), \\
\text { trace of } u \text { on } \partial D=\varphi_{0} .
\end{array}\right.
$$

Our result is the following. 
Theorem 2.1. Let $1<p<2$ and $\varphi_{0}, \varphi_{1}$ be of class $C^{\infty}$ on $\partial D$ and such that:

(i) $\int_{\partial D} \varphi_{1} d s=0$;

(ii) $\int_{\partial D} n_{2}\left(\varphi_{1}-\varphi_{0}\right) d s=0$.

Then there exist a linear second-order uniformly elliptic operator $L$ in divergence form, with bounded measurable coefficients, and a function $u \in W^{1, p}(D)$, which is a solution of (1.2) (that is, satisfies (2.1)).

Proof. Let $\left(\psi_{0}, \psi_{1}\right)$ be the solution on $\partial D$ of the system

$$
\left\{\begin{array}{l}
n_{1} \psi_{1}-n_{2} \frac{d \psi_{0}}{d \theta}=\varphi_{0}, \\
\frac{d}{d \theta}\left(n_{2} \psi_{1}+n_{1} \frac{d \psi_{0}}{d \theta}\right)=-\varphi_{1} .
\end{array}\right.
$$

The functions $\psi_{0}$ and $\psi_{1}$ exist and are regular on $\partial D$ by the hypotheses on $\varphi_{0}$, $\varphi_{1}$. In particular, condition (ii) is equivalent to the condition $\int_{\partial D}\left(d \psi_{0} / d \theta\right) d s=0$ by integration by parts.

By [1, Theorem 3.3], there exist $v \in W^{2, p}(D)$ and a second-order uniformly elliptic operator in nondivergence form and with bounded measurable coefficients, $\bar{L}:=$ $\widetilde{a}^{11} \partial_{x x}+2 \widetilde{a}^{12} \partial_{x y}+\widetilde{a}^{22} \partial_{y y}$, such that

$$
\left\{\begin{array}{l}
\widetilde{L} v=0 \\
\left.v\right|_{\partial D}=\psi_{0}, \\
\left.\frac{\partial v}{\partial n}\right|_{\partial D}=\psi_{1} .
\end{array} \text { in } D,\right.
$$

Let $u=v_{x} \in W^{1, p}(D)$. The equation $\widetilde{L} v=0$ can be written as

$$
\frac{\widetilde{a}^{11}}{\widetilde{a}^{22}} v_{x x}+2 \frac{\widetilde{a}^{12}}{\widetilde{a}^{22}} v_{x y}+v_{y y}=0 \quad \text { a.e. in } D,
$$

and, by formally differentiating with respect to $x$,

$$
L u=\left(a^{11} u_{x}\right)_{x}+\left(a^{12} u_{y}\right)_{x}+u_{y y}=0,
$$

where $a^{11}=\widetilde{a}^{11} / \widetilde{a}^{22}$ and $a^{12}=2 \widetilde{a}^{12} / \widetilde{a}^{22}$. According to our definition, $u \in W^{1, p}$ is a solution to (1.2) for the operator $L$ defined in (2.3) if and only if the trace of $u$ on $\partial D$ is equal to $\varphi_{0}$ and

$$
\int_{D}\left(a^{11} u_{x} \eta_{x}+a^{12} u_{y} \eta_{x}+u_{y} \eta_{y}\right) d x d y=\int_{\partial D} \varphi_{1} \eta d s
$$

for any $\eta \in C^{1}(\bar{D})$. On the other hand,

$$
\begin{aligned}
\int_{D}\left(a^{11} u_{x} \eta_{x}+a^{12} u_{y} \eta_{x}+u_{y} \eta_{y}\right) d x d y \\
\quad=\int_{D} \frac{\tilde{a}^{11} v_{x x} \eta_{x}+2 \tilde{a}^{12} v_{x y} \eta_{x}+\tilde{a}^{22} v_{x y} \eta_{y}}{\tilde{a}^{22}} d x d y
\end{aligned}
$$




$$
\begin{aligned}
& =\int_{D}\left(\frac{\tilde{a}^{11} v_{x x}+2 \tilde{a}^{12} v_{x y}+\tilde{a}^{22} v_{y y}}{\tilde{a}^{22}} \eta_{x}+\left(v_{x y} \eta_{y}-v_{y y} \eta_{x}\right)\right) d x d y \\
& =\int_{D}\left(v_{x y} \eta_{y}-v_{y y} \eta_{x}\right) d x d y .
\end{aligned}
$$

If we consider a sequence $v_{n} \in C^{3}(\bar{D})$ converging to $v$ in $W^{2, p}(D)$, then

$$
\int_{D}\left(v_{x y} \eta_{y}-v_{y y} \eta_{x}\right) d x d y=\lim _{n \rightarrow \infty} \int_{D}\left(\left(v_{n}\right)_{x y} \eta_{y}-\left(v_{n}\right)_{y y} \eta_{x}\right) d x d y .
$$

However,

$$
\begin{aligned}
\int_{D}\left(\left(v_{n}\right)_{x y} \eta_{y}-\left(v_{n}\right)_{y y} \eta_{x}\right) d x d y & =\int_{D}\left(\left(\left(v_{n}\right)_{x y} \eta\right)_{y}-\left(\left(v_{n}\right)_{y y} \eta\right)_{x}\right) d x d y \\
& =\int_{\partial D}\left(n_{2}\left(v_{n}\right)_{x y}-n_{1}\left(v_{n}\right)_{y y}\right) \eta d s \\
& =-\int_{\partial D}\left(\frac{d\left(v_{n}\right)_{y}}{d \theta}\right) \eta d s=\int_{\partial D}\left(v_{n}\right)_{y} \frac{d \eta}{d \theta} d s
\end{aligned}
$$

and

$$
\lim _{n \rightarrow \infty} \int_{\partial D}\left(v_{n}\right)_{y} \frac{d \eta}{d \theta} d s=\int_{\partial D} v_{y} \frac{d \eta}{d \theta} d s
$$

Moreover, since

$$
\left.v\right|_{\partial D}=\psi_{0} \in C^{\infty}(\partial D) \quad \text { and }\left.\quad \frac{\partial v}{\partial n}\right|_{\partial D}=\psi_{1} \in C^{\infty}(\partial D)
$$

we have also that

$$
\left.v_{y}\right|_{\partial D}=\left.n_{2} v_{r}\right|_{\partial D}+\left.n_{1} v_{\theta}\right|_{\partial D} \in C^{\infty}(\partial D)
$$

and

$$
\int_{\partial D} v_{y} \frac{d \eta}{d \theta} d s=-\int_{\partial D} \frac{d v_{y}}{d \theta} \eta d s .
$$

This implies that

$$
\int_{D}\left(a^{11} u_{x} \eta_{x}+a^{12} u_{y} \eta_{x}+u_{y} \eta_{y}\right) d x d y=-\int_{\partial D} \frac{d v_{y}}{d \theta} \eta d s,
$$

which means that $u$ solves

$$
\begin{cases}L u=0 & \text { a.e. in } D \\ \left.\frac{\partial u}{\partial N}\right|_{\partial \Omega}=-\frac{d v_{y}}{d \theta} & \end{cases}
$$

Since, from (2.2),

$$
\begin{gathered}
\left.u\right|_{\partial D}=\left.v_{x}\right|_{\partial D}=n_{1} \psi_{1}-n_{2} \frac{d}{d \theta} \psi_{0}=\varphi_{0}, \\
\left.\frac{\partial u}{\partial N}\right|_{\partial D}=-\frac{d v_{y}}{d \theta}=-\frac{d}{d \theta}\left(n_{2} \psi_{1}+n_{1} \frac{d \psi_{0}}{d \theta}\right)=\varphi_{1},
\end{gathered}
$$

it follows immediately that $u$ is a solution to (1.2) with $L$ defined in (2.3). 


\section{References}

[1] C. Giannotti and P. Manselli, 'On elliptic extensions in the disk', Potential Anal. 33 (2010), 249-262.

[2] O. A. Ladyzhenskaya and N. N. Ural'tseva, Linear and Quasilinear Elliptic Equations (Academic Press, New York, 1968).

[3] T. H. Wolff, 'Some constructions with solutions of variable coefficient elliptic equations', J. Geom. Anal. 3 (1993), 423-511.

ORAZIO ARENA, Dipartimento di Costruzioni e Restauro, Università di Firenze, Piazza Brunelleschi 6, I-50121 Firenze, Italy

e-mail: arena@unifi.it

CRISTINA GIANNOTTI, Scuola di Scienze e Tecnologie, Università di Camerino, Via Madonna delle Carceri, I- 62032 Camerino (Macerata), Italy

e-mail: cristina.giannotti@unicam.it 